\title{
Lack of Association of FLT3 rs2504235 and Absence of SLITRKI var32I in Patients with Tic Disorders from Guangdong Province, China
}

\author{
Ming Gao', Haisheng $\mathrm{Lin}^{2}$, Bingxiao $\mathrm{Li}^{3}$, Junjie Wen $\mathbb{D}^{3}$, Yingying Wang', Zhanhui Zhang ${ }^{4} *$, \\ Wenxiong Chen ${ }^{2} *$
}

\begin{abstract}
'Department of Neurology, First Affiliated Hospital of Jinan University, Guangzhou, Guangdong, 510630, People's Republic of China; ${ }^{2}$ Department of Neurology, Guangzhou Women and Children's Medical Center, Guangzhou Medical University, Guangzhou, Guangdong, 5I0I20, People's Republic of China; ${ }^{3}$ Department of Pediatrics, First Affiliated Hospital, Jinan University, Guangzhou, Guangdong, 510630, People's Republic of China; ${ }^{4}$ Clinical Medicine Research Institute, First Affiliated Hospital, Jinan University, Guangzhou, 510630, People's Republic of China
\end{abstract}

*These authors contributed equally to this work

Correspondence: Zhanhui Zhang, First Affiliated Hospital, Jinan University, 613 West Huangpu Avenue, Tianhe, Guangzhou 510630, Guangdong, People's Republic of China, Tel +86-20-3868-8749, Email zzhaddress@I26.com

\begin{abstract}
Objective: Tic disorders (TDs) are highly polygenic and heritable neurodevelopmental disorders characterized by the presence of movements (motor tics) and/or vocalizations (phonic tics). SLITRK1 is a pathogenic variation of TD, and in a recent genome-wide association study in those of European ancestry, a single-nucleotide polymorphism (rs2504235) in the FLT3 gene was significantly associated with TDs/Tourette's syndrome. However, these results need to be proved in different populations. This study aimed to determine whether these two genetic variants were also associated with TD patients in south China.

Methods: A total of 116 child TD patients and 114 healthy controls were included. All children underwent peripheral blood sampling for genomic DNA extraction. Gene fragments with two single-nucleotide polymorphisms were amplified by PCR and sequenced by Sanger chain termination before genotype analysis.

Results: SLITRK1 var321 was not observed in any of the TD patients or controls. No significant difference was observed in allelic frequencies or genotypic distributions of rs2504235 between TD patients and controls.

Conclusion: Our results provide no evidence to support the previous conclusion that SLITRK1 var321 plays a major role in TDs, and FLT3 rs2504235 was not significantly associated with TDs in our cohort.
\end{abstract}

Keywords: tic disorders, Tourette's syndrome, SLITRK1, SLIT and NTRK like family member 1, FLT3, Fms related receptor tyrosine kinase 3

\section{Introduction}

Tic disorders (TDs) are childhood-onset neurodevelopmental disorders characterized by sudden, rapid, recurrent, arrhythmic movements (motor tics) and/or vocalizations (phonic tics). TDs are more common in boys, with a male: female ratio of 3-4:1. ${ }^{1,2}$ Tics most commonly involve the head, neck, and upper body, most begin before the age of 18 years, and they can be suppressed for variable periods. ${ }^{3}$ TDs typically have an onset at age 6-8 years, ${ }^{4}$ and the mean age of peak tic severity is $10 \pm 2.4$ years. ${ }^{5,6}$

In the DSM- $5,{ }^{6}$ ICD-10, ${ }^{7}$ and CCMD- $3,{ }^{8}$ TDs are classified into three types: transient/provisional TD (TTD/PTD), chronic TD (CTD), and Tourette's syndrome (TS). CTD is then divided into chronic motor TD (CMTD) and chronic phonic TD (CPTD). According to existing classifications, TS varies from CMTD in only one way: in TS, it is a requirement that both multiple motor tics and at least one phonic tic be present, while in CMTD it is required that only motor tics be present. ${ }^{6,7}$

Recent studies have estimated that the prevalence of TS in children in the general population is $0.3 \%-0.9 \%,{ }^{1,9-11}$ while that of CMTD is $0.5 \%-1.65 \%{ }^{1,9-11}$ Due to this additional criterion regarding the added presence of phonic tics, TS 
in the community is rarer than CMTD. ${ }^{12,13}$ Accordingly, the prevalence of PTD, the mildest form of all primary TDs is much higher $-5 \%-47 \%{ }^{14}$ TD is often comorbid with other psychiatric conditions, mainly attention-deficit/hyperactivity disorder (ADHD), obsessive-compulsive disorder (OCD) and non-OCD anxiety disorders, mood disorders, disruptive behavior disorders, pervasive developmental disorders, and problems with sleep. ${ }^{15-18}$

Several decades of investigation have confirmed that TDs are highly polygenic and heritable, with multiple genetic variants widely distributed throughout the genome. Multiple candidate genes (eg, DRD2, DRD4, 5-HT2C, SERTSLITRK1, IMMP2L, CNTNAP2, HDC, PNKD, and NLGN4) in multiple neural systems, including dopaminergic, serotonergic, and histaminergic pathways, have been identified through linkage studies and structural genomic aberrations, in which very rare genetic variants with large effects were found in TD patients and families. ${ }^{14,19-22}$

Recently, genome-wide association studies (GWASs) and gene-based analyses of the largest samples thus far of TD/ TS patients of European ancestry showed that the FLT3 gene was significantly associated postcorrection with TDs on 18,079 gene tests $\left(P=8.9 \times 10^{-7}\right)$. The most significant single-nucleotide polymorphism (SNP) in the FLT3 locus, rs2504235 (ENST00000241453.12:c.1206-1461T >C), was the only SNP to surpass the GW significance threshold (OR 1.16, $P=2.1 \times 10^{-8}$ ) on primary meta-analysis and was significantly associated with FLT3 expression in both the cerebellum $\left(P=6.5 \times 10^{-10}\right)$ and cerebral cortex $\left(P=2.6 \times 10^{-11}\right) .^{23}$

We are interested in whether rs 2504235 has a significant association with TDs in Chinese, so we screened it in TD patients of Guangdong Province China. We also screened another pathogenic mutation in the 3'UTR of SLITRK1 var321 (ENST00000674365.1:c.*689G>T), which alters the binding of SLITRK1 mRNA to microRNA hsa-miR189 and affects neurite outgrowth. ${ }^{24}$ Several replication studies have investigated the involvement of SLITRK1 var321 in TS pathogenesis, though its role has not been elucidated. ${ }^{25-33}$ In this study, 116 patients with TDs were investigated to explore the potential role of SLITRK1 var321 and FLT3 in disease pathogenesis.

\section{Methods}

\section{Subjects}

A total of 116 unrelated children (93 boys and 23 girls) with TDs were enrolled in this study. Of these, 33 had only TS, 33 only TTD, and 50 only CTD. They were evaluated at Guangzhou Women and Children's Medical Center and the First Affiliated Hospital of Jinan University, Guangzhou. A total of 114 unrelated healthy control subjects matched in age and sex were recruited from individuals who underwent a regular health examination during the same period as the control group. We implemented this study design and all related procedures in accordance with the Declaration of Helsinki. This study was approved by the Ethics Committee of Guangzhou Women and Children's Medical Center. All parents signed an informed-consent document before blood tests were performed. TD diagnosis followed the criteria of the DSM-5, ${ }^{6}$ ICD-10, ${ }^{7}$ and CCMD-3. ${ }^{8}$ The age at which first tics presented was $2-15(7.61 \pm 2.51)$. All children underwent peripheral blood sampling for genotype analysis.

\section{Genetic Analysis}

Genomic DNA was prepared from peripheral blood using a whole-blood genomic DNA minikit (SimGen, Hangzhou, China). PCR tests were performed with a total volume of $30 \mu \mathrm{L}$ containing 50-100 ng genomic DNA, $0.6 \mu \mathrm{L}$ of each 10 $\mu \mathrm{M}$ primer (Table 1), $12.5 \mu \mathrm{L}$ premix Taq (Takara Taq version 2.0). PCR amplification was performed in a programmable thermal cycler system (Eppendorf Mastercycler nexus). Cycling conditions for PCRs were set at one cycle at $94^{\circ} \mathrm{C}$ for 5 minutes, 35 cycles at $94^{\circ} \mathrm{C}$ for 30 seconds, $62^{\circ} \mathrm{C}$ for 30 seconds, and $72^{\circ} \mathrm{C}$ for 40 seconds, and one final cycle for extension at $72^{\circ} \mathrm{C}$ for 10 minutes. PCR products were loaded into $2 \%$ agarose gel containing GoldView (Solarbio) for

Table I Primers for FLT3 and SLITRKI

\begin{tabular}{|l|c|c|}
\hline Fragment & Forward $\left(\mathbf{5}^{\prime} \rightarrow \mathbf{3}^{\prime} \mathbf{)}\right.$ & Reverse $\left.\mathbf{( 5}^{\prime} \rightarrow \mathbf{3}^{\prime}\right)$ \\
\hline FLT3 & GCAGCCCTATGACTTCCCGT & GGTTCACCGTGTTAGCCAGG \\
SLITRKI var32I & CTCTTACCTGATAAGTTCCATCG & GCAGCCTAAGCACTAGAGTGAC \\
\hline
\end{tabular}


electrophoresis. Amplified DNA fragments were sent to Guangzhou Tianyihuiyuan Gene Technology for Sanger sequencing (Figure 1).

\section{Statistical Methods}

SPSS 20.0 was used for data analysis. Genotype and allele frequencies are expressed as percentages. The fitness of the genotype and allele was tested using the Hardy-Weinberg equilibrium test, ${ }^{34,35}$ and polymorphism differences between TD patients and control groups were analyzed using single-marker $\chi^{2}$ tests. The genotype quality controls were minor-allele frequency (MAF) $>0.1$, Hardy-Weinberg equilibrium $>0.001$, and call ratio $>95 \%$. The threshold for all statistical tests was set as 0.05 .

\section{Results}

\section{Genetic Distribution}

The Hardy-Weinberg equilibrium results showed that the genetic distribution of all subjects reached equilibrium $(P=0.199, P>0.05$; Table 2), proving that the experimental population came from the same Mendelian population.

\section{Baseline Clinical Characteristics}

The male:female ratio for the cohort was 4.04:1. Mean age at onset was 7.61 \pm 2.51 years, with mean age of boys (7.59 \pm 2.56 years) similar to girls $(7.70 \pm 2.36 ; P=0.859)$. Mean age of TTD patients ( $6.67 \pm 2.38$ years) was significantly lower than the mean age of CTD $(7.98 \pm 2.46 ; P=0.019)$ and TS $(8.00 \pm 2.54 ; P=0.030)$. Of the 54 children aged $>6$ years who underwent psychological evaluation, 24 were diagnosed with ADHD (TTD 5, CTD 9, TS 10). Five cases of TTD, seven of CTD, and eleven of TS were assessed for mood, including five cases of combined anxiety (CTD 2, TS 3), three of depression (CTD 1, TS 2), and six of compulsion (TTD 1, CTD 1, TS 4).

\section{Association Between rs2504235 and TD}

Genotypic distributions of rs2504235 within FLT3 were $63.79 \%(\mathrm{C} / \mathrm{C}), 35.34 \%(\mathrm{C} / \mathrm{T})$, and $0.87 \%(\mathrm{~T} / \mathrm{T})$. Mean age of C/ $\mathrm{T}(8.37 \pm 2.70)$ patients was significantly older than the mean age of $\mathrm{C} / \mathrm{C}(7.24 \pm 2.30)$ patients $(P=0.020)$. In children with TDs, we found no association between TD classification, genotypic distribution of rs2504235, or sex ( $P>0.05)$. No significant differences were observed in allelic frequencies or genotypic distribution of rs 2504235 between patients and controls $(P=0.157, P=0.199$ ). MAF of rs 2504235 was 0.185 (43 of 232) in the TD cohort and 0.189 (43 of 228) in controls, with no significant difference $(P>0.05)$. Furthermore, we did not observe any novel nonsynonymous variation in the region covered. Results of association analyses between patients and controls are shown in Table 3 .

\section{Association Between SLITRKI var32I and TDs}

Genotypic distribution of SLITRK1 var321 (NM_052910.2:c.*689G>A) was 100 (G/G) among the 116 patients with TDs. This suggested that SLITRK1 var321 was not a major component of the pathogenesis of TDs.

\section{Discussion}

Only two GWASs on TS have been published, ${ }^{23,36}$ with no SNPs meeting the criteria for GW significance $\left(p<5 \times 10^{-8}\right)$ and one reaching marginal GW significance (rs7868992, located in an intronic region of COL27A1; $P=1.85 \times 10^{-6}$ ) in 1,285 cases and 4,964 ancestry-matched controls of European ancestry, including two European-derived population isolates. ${ }^{36}$ The most recent TS GWAS ${ }^{23}$ with the largest sample (4,819 cases, 9,488 controls) identified one significant GW locus within FLT3 on chromosome 13, rs 2504235 (OR 1.16, $P=2.13 \times 10^{-8}$ ). It was also significantly associated with FLT3 expression in both in the cerebellum and cerebral cortex. ${ }^{23}$ FLT3, a member of the type III receptor tyrosinekinase family, is expressed almost exclusively in the hematopoietic compartment. FLT3 induces dimerization and activation of its intrinsic tyrosine-kinase activity. Activation of FLT3 mediates cell survival, cell proliferation, and differentiation of hematopoietic progenitor cells. FLT3 activity has been implicated in several diseases, most prominently acute myeloid leukemia, where around a third of patients carry an activating mutant of FLT3. Overactivity of FLT3 has also been implicated in autoimmune diseases, such as rheumatoid arthritis. ${ }^{37}$ 


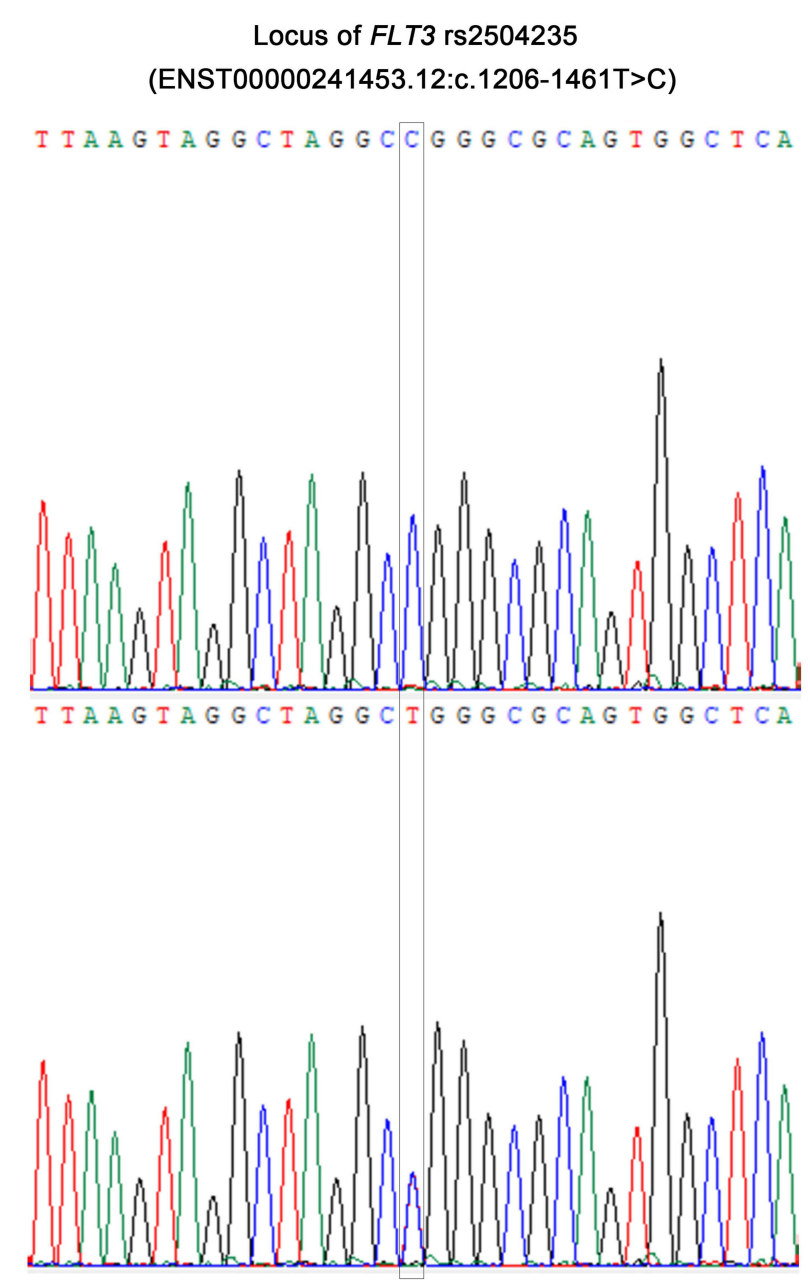

Locus of SLITRK1 var321

(ENST00000674365.1:c.*689G>T)

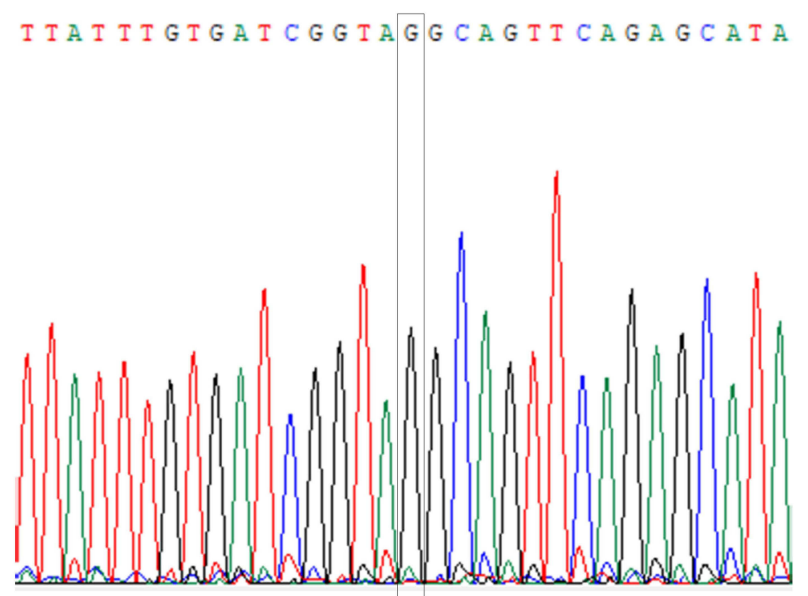

Figure I Sanger sequencing chromatograms of the targeted DNA fragments: locus of FLT3 rs2504235 and SLITRKI var32I.

Our results showed that there was no SNP-frequency difference between TD patients and controls, similar to the results of replication research on rs 2504235 with significant GW associations in an independent case-control replication sample from Iceland (706 cases, 6,068 controls). ${ }^{23}$ One of the possible reasons is the genetic background and population 
Table 2 Hardy-Weinberg equilibrium test results

\begin{tabular}{|l|c|c|c|c|c|c|}
\hline \multirow{2}{*}{} & \multirow{2}{*}{$\mathbf{n}$} & \multicolumn{2}{|c|}{ Genotypic distribution of rs2504235 } & \multicolumn{2}{c|}{ Allele frequency of rs2504235 } \\
\cline { 3 - 7 } & & G/G & G/A & A/A & G & A \\
\hline Actual frequency & 116 & $74(63.79 \%)$ & $41(35.34 \%)$ & I (0.87\%) & $189(81.47 \%)$ & $43(18.53 \%)$ \\
Theoretical frequency & 116 & $76.99(66.37 \%)$ & $17.52(15.10 \%)$ & $3.98(3.43 \%)$ & & \\
\hline
\end{tabular}

Table 3 Association analysis of patients and controls

\begin{tabular}{|c|c|c|c|c|c|c|c|}
\hline \multirow[t]{2}{*}{ SNP } & \multirow[t]{2}{*}{ Allele (major/minor) } & \multicolumn{3}{|c|}{ Minor-allele frequency } & \multicolumn{3}{|c|}{ Genotypic distribution $^{a}$ of rs 2504235} \\
\hline & & Patients & Controls & $P^{\mathbf{b}}$ & Patients & Controls & $P^{\mathbf{b}}$ \\
\hline rs 2504235 & G/A & 0.185 & 0.189 & 0.157 & $74 / 41 / 1$ & $77 / 31 / 6$ & 0.199 \\
\hline
\end{tabular}

Notes: ${ }^{a}$ Number of participants (major homo/hetero/minor homo); ${ }^{b}$ patients vs controls $\left(\chi^{2}\right.$ test).

Abbreviation: SNP, single-nucleotide polymorphism.

characteristics between Chinese and Europeans. In data from the China Metabolic Analytics Project, the MAF of rs 2504235 is 0.179 (http://www.mbiobank.com/search/?searchContent=rs2504235), close to our results of 0.185 (43 of 232 ) in the TD cohort, 0.189 (43 of 228) in controls, and 0.168 in East Asians in the 1,000 Genomes Project. This MAF value was lower than the 0.38 obtained in a second GWAS. ${ }^{23}$ Another possible reason is that $\mathrm{rs} 2504235$ has no significant association with FLT3 expression in the Chinese population. Unfortunately, we cannot collect expressionlevel data on genes, including FLT3, in the brains of Chinese TD patients. As we know, there have been no reports on FLT3 being associated with any TD and its expression and function in brain tissue. Consequently, future larger-scale GWASs in the Chinese population should aid in identifying individual genes underlying susceptibility to TS.

In 2005, Abelson et al screened 174 unrelated patients with TS and identified a noncoding missense variant (ENST00000674365.1:c. ${ }^{*} 689 \mathrm{G}>\mathrm{T}$, or c. $2977+2067 \mathrm{G}>\mathrm{A}$ ), named var321, in the $3^{\prime} \mathrm{UTR},{ }^{24}$ and SLITRK1 was considered one of TS candidate genes. Since that initial study ${ }^{24}$ several replication studies have investigated the involvement of SLITRK1 var321 in TS pathogenesis, but with complex results. Several studies did not find any involvement. ${ }^{25-33}$ However, in a study on Ashkenazi Jewish patients with TS, five unrelated parents of Ashkenazi Jewish ancestry had var321, and only three transmitted it to their children. ${ }^{38}$ In addition, a study on screened samples of TS patients and their families found var321 in parents of two unrelated TS probands, but no transmission of the variant to probands. Only one of those parents was diagnosed with TS. As such, the prevalence of var321 in all TS individuals was $0.1 \%$ (one of 1,048). ${ }^{39}$ In this study, we did not observe SLITRK1 var321 in the investigated TD cohort. However, considering our small study sample and the low MAF of these two variants, it is necessary to expand the sample size to further evaluate whether SLITRK1 var321 and FLT3 rs2504235 variants are related to TD in the Chinese population.

\section{Conclusion}

Our results suggest that SLITRK1 var321 is unlikely to play a major role in TD in the Chinese population. FLT3 rs2504235 was not significantly associated with TD in our cohort.

\section{Acknowledgments}

We thank all participants who took part in this project. This study got financial support from the National Natural Science Foundation of China (81670813) and Medical Science and Technology Research Foundation of Guangdong Province (B2019030).

\section{Disclosure}

The authors report no potential conflicts of interest in this work. 


\section{References}

1. Knight T, Steeves T, Day L, et al. Prevalence of tic disorders: a systematic review and meta-analysis. Pediatr Neurol. 2012;47(2):77-90. doi:10.1016/j.pediatrneurol.2012.05.002

2. Freeman RD. Tic disorders and ADHD: answers from a world-wide clinical dataset on Tourette syndrome. Eur Child Adolesc Psychiatry. 2007;16:15-23. doi:10.1007/s00787-007-1003-7

3. American Psychiatric Association. Diagnostic and Statistical Manual of Mental Disorders: DSM-IVTR. 4th ed. Washington, DC: American Psychiatric Association; 2000.

4. Scahill L, Sukhodolsky DG, Williams SK, et al. Public health significance of tic disorders in children and adolescents. Adv Neurol. 2005;96:240-248.

5. Leckman JF, Zhang H, Vitale A, et al. Course of tic severity in Tourette syndrome: the first two decades. Pediatrics. 1998;102(1 Pt 1):14-19. doi:10.1542/peds.102.1.14

6. American Psychiatric Association. Diagnostic and Statistical Manual of Mental Disorders. 5th ed. Washington (DC): American Psychiatric Association; 2013.

7. World Health Organisation. International statistical classifcation of diseases and related health problems, 10th revision (ICD-10). Geneva: WHO; 1992.

8. Chinese Neurology Society, Chinese Medical Association. Classification and Diagnostic Criteria of Mental Disorders in China. 3rd ed. Jinan: Shandong Science and Technology Press; 2001:9-125.

9. Kraft JT, Dalsgaard S, Obel C, et al. Prevalence and clinical correlates of tic disorders in a community sample of school-age children. Eur Child Adolesc Psychiatry. 2012;21(1):5-13. doi:10.1007/s00787-011-0223-z

10. Scharf JM, Miller LL, Mathews CA, et al. Prevalence of Tourette syndrome and chronic tics in the population-based Avon longitudinal study of parents and children cohort. J Am Acad Child Adolesc Psychiatry. 2012;51(2):192-201. doi:10.1016/j.jaac.2011.11.004

11. Scharf JM, Miller LL, Gauvin C, et al. Population prevalence of Tourette syndrome: a systematic review and meta-analysis. Mov Disord. 2015;30 (2):221-228. doi: 10.1002/mds.26089

12. Khalifa N, Von Knorring A. Prevalence of tic disorders and Tourette syndrome in a Swedish school population. Dev Med Child Neurol. 2003;45 (5):315-319. doi:10.1111/j.1469-8749.2003.tb00402.x

13. Spencer TJ, Biederman J, Harding M, et al. The relationship between tic disorders and tourette's syndrome revisited. J Am Acad Child Adolesc Psychiatry. 1995;34(9):1133-1139. doi:10.1097/00004583-199509000-00009

14. Black KJ, Black ER, Greene DJ, et al. Provisional tic disorder: what to tell parents when their child first starts ticcing. F1000Res. 2016;5:696. doi:10.12688/f1000research.8428.1

15. Hirschtritt ME, Lee PC, Pauls DL, et al. Lifetime prevalence, age of risk, and genetic relationships of comorbid psychiatric disorders in Tourette syndrome. JAMA Psychiatry. 2015;72(4):325-333. doi:10.1001/jamapsychiatry.2014.2650

16. Freeman RD, Fast DK, Burd L, et al. An international perspective on Tourette syndrome: selected findings from 3500 individuals in 22 countries. Dev Med Child Neurol. 2000;42(7):436-447. doi:10.1017/S0012162200000839

17. Cavanna AE, Servo S, Monaco F, et al. The behavioral spectrum of Gilles de la Tourette syndrome. J Neuropsychiatry Clin Neurosci. 2009;21 (1):13-23. doi:10.1176/jnp.2009.21.1.13

18. Robertson MM. A personal 35 year perspective on Gilles de la Tourette syndrome: prevalence, phenomenology, comorbidities, and coexistent psychopathologies. Lancet Psychiatry. 2015;2(1):68-87. doi:10.1016/S2215-0366(14)00132-1

19. Pauls DL, Fernandez TV, Mathews CA, et al. The inheritance of Tourette disorder: a review. J Obsessive Compuls Relat Disord. 2014;3 (4):380-385. doi:10.1016/j.jocrd.2014.06.003

20. Huang AY, Yu D, Davis LK, et al. Rare copy number variants in NRXN1 and CNTN6 increase risk for Tourette syndrome. Neuron. 2017;94 (6):1101-1111.e7. doi:10.1016/j.neuron.2017.06.010

21. Willsey AJ, Fernandez TV, Yu D, et al. De novo coding variants are strongly associated with Tourette disorder. Neuron. 2017;94(3):486-499.e9. doi:10.1016/j.neuron.2017.04.024

22. Wang S, Mandell JD, Kumar Y, et al. De novo sequence and copy number variants are strongly associated with Tourette disorder and implicate cell polarity in pathogenesis. Cell Rep. 2018;24(13):3441-3454.e12. doi:10.1016/j.celrep.2018.08.082

23. Yu D, Sul JH, Tsetsos F, et al. Interrogating the genetic determinants of Tourette's syndrome and other tic disorders through genome-wide association studies. Am J Psychiatry. 2019;176(3):217-227. doi:10.1176/appi.ajp.2018.18070857

24. Abelson JF, Kwan KY, Oroak BJ, et al. Sequence variants in SLITRK1 are associated with Tourette's syndrome. Science. 2005;310(5746):317-320. doi:10.1126/science. 1116502

25. De Miranda DM, Wigg K, Kabia EM, et al. Association of SLITRK1 to Gilles de la Tourette syndrome. Am J Med Genet B Neuropsychiatr Genet. 2009;150B(4):483-486. doi:10.1002/ajmg.b.30840

26. Deng H, Le W, Xie WJ, et al. Examination of the SLITRK1 gene in Caucasian patients with Tourette syndrome. Acta Neurol Scand. 2006;114 (6):400-402. doi:10.1111/j.1600-0404.2006.00706.x

27. Verkerk AJ, Cath DC, Der Linde HV, et al. Genetic and clinical analysis of a large Dutch Gilles de la Tourette family. Mol Psychiatry. 2006;11 (10):954-964. doi:10.1038/sj.mp.4001877

28. Chou IC, Wan L, Liu SC, et al. Association of the Slit and Trk-like 1 gene in Taiwanese patients with Tourette syndrome. Pediatr Neurol. 2007;37 (6):404-406. doi:10.1016/j.pediatrneurol.2007.06.017

29. Fabbrini G, Pasquini M, Aurilia C, et al. A large Italian family with Gilles de la Tourette syndrome: clinical study and analysis of the SLITRK1 gene. Mov Disord. 2007;22(15):2229-2234. doi:10.1002/mds.21697

30. Orth M, Djarmati A, Baumer T, et al. Autosomal dominant myoclonus-dystonia and Tourette syndrome in a family without linkage to the SGCE gene. Mov Disord. 2007;22(14):2090-2096. doi:10.1002/mds.21674

31. Zimprich A, Hatala K, Riederer F, et al. Sequence analysis of the complete SLITRK1 gene in Austrian patients with Tourette's disorder. Psychiatr Genet. 2008;18(6):308-309. doi:10.1097/YPG.0b013e3283060f6f

32. Yasmeen S, Melchior L, Bertelsen B, et al. Sequence analysis of SLITRK1 for var321 in Danish patients with Tourette syndrome and review of the literature. Psychiatr Genet. 2013;23(3):130-133. doi:10.1097/YPG.0b013e328360c880 
33. Inai A, Tochigi M, Kuwabara H, et al. Analysis of SLITRK1 in Japanese patients with Tourette syndrome using a next-generation sequencer. Psychiatr Genet. 2015;25(6):256-258. doi:10.1097/YPG.0000000000000104

34. Hardy GH Mendelian proportions in a mixed population. Classic papers in genetics. Englewood Cliffs, NJ: Prentice-Hall, Inc.; 1908:60-62.

35. Weinberg W. ber den Nachweis der Vererbung beim Menschen. Jahreshefte Des Vereins Für Vaterländische Naturkunde in Württemberg [On the proof of heredity in humans. - Annual booklet of the association for patriotic natural history in Württemberg]. 1908;64:369-382. German.

36. Scharf JM, Yu D, Mathews CA, et al. Genome-wide association study of Tourette's syndrome. Mol Psychiatry. 2013;18(6):721-728. doi:10.1038/ mp.2012.69

37. Kazi JU, Ronnstrand L. FMS-like tyrosine kinase 3/FLT3: from basic science to clinical implications. Physiol Rev. 2019;99(3):1433-1466. doi:10.1152/physrev.00029.2018

38. Keenkim D, Mathews CA, Reus VI, et al. Overrepresentation of rare variants in a specific ethnic group may confuse interpretation of association analyses. Hum Mol Genet. 2006;15(22):3324-3328. doi:10.1093/hmg/ddl408

39. Scharf JM, Moorjani P, Fagerness J, et al. Lack of association between SLITRK1var321 and Tourette syndrome in a large family-based sample. Neurology. 2008;70(16 Pt 2):1495-1496. doi:10.1212/01.wnl.0000296833.25484.bb

Neuropsychiatric Disease and Treatment

\section{Publish your work in this journal}

Neuropsychiatric Disease and Treatment is an international, peer-reviewed journal of clinical therapeutics and pharmacology focusing on concise rapid reporting of clinical or pre-clinical studies on a range of neuropsychiatric and neurological disorders. This journal is indexed on PubMed Central, the 'PsycINFO' database and CAS, and is the official journal of The International Neuropsychiatric Association (INA). The manuscript management system is completely online and includes a very quick and fair peer-review system, which is all easy to use. Visit http://www.dovepress.com/testimonials.php to read real quotes from published authors.

Submit your manuscript here: https://www.dovepress.com/neuropsychiatric-disease-and-treatment-journal 